\title{
Research on Evaluation Method of Contiguous Sense of Linen Fabrics
}

\author{
Caiyuan Kuang ${ }^{12}$ \\ ${ }^{1}$ College of Art \\ Jinling Institute of Technology \\ Nanjing, China \\ E-mail: kcy@jit.edu.cn \\ ${ }^{2}$ College of Textile and Clothing Engineering \\ Soochow University \\ Suzhou, China \\ E-mail: kcy@jit.edu.cn
}

\begin{abstract}
Objective: To test the feasibility of the proposed method that evaluates the contiguous sense of linen fabrics by using experimental paradigm of behavioral experiment. (2) Methods: The experimental program is written with E-Prime 2.0, while the expression of the experimental stimulus material is realized with the self-made contiguous sense measurement device, which output the senses of itch, roughness and smoothness among the fabric contiguous sense. (3) Results: The evaluation values of the senses of urtication, roughness and smoothness differs significant, and can distinguish the intensity of the contiguous senses of 8 fabrics fully. There is no obvious difference among the evaluation methods of contiguous senses of those fabrics by using measurement device or manual measurement. (4) Conclusion: The self-made contiguous sense measurement device combined with the introduction of behavioral experimental paradigm is feasible, presenting the contiguous sense of linen fabrics effectively.
\end{abstract}

Keywords- Contiguous Sen se; Fabric; Evaluation Method; Evaluation Device; Perception

\section{INTRODUCTION}

As customers' needs of sensible clothing have drastically increased, research for clothing design considering visual, auditory, and tactile sensibilities has been actively conducted. As for tactile sensibility, varieties of senses arise when skin contacts fabric. The sense of urtication arising fro $\mathrm{m}$ fabric has been proven as one of the most discomforting tactile sensibility [1]. The sense of roughness [2], refers to the sensibility of smoothness or roughness experienced when skin contacts fabric surface, which is a kind of physiological and psychic combined reaction arising fro $m$ the stimulation formed by the surface topography and texture features of fabric.

Subjective evaluation is a common method to estimate urtication of fabrics [3]. However, this method needs to improve its efficiency in the administration of an experiment and the management of evaluation data. For example, the questionnaire method lacks efficiency in terms of time and staffing because the administrator must present specimens in person and handle evaluation data manually. Furthermore, the individual differences of subjects are easy to bring about instability to the eventual evaluation of urtication sense.

There are several objective evaluation ways, such as pallial marking method, voice frequency device measurement method, laser counting to highlight the quantity of hairiness, and low stress compression measurement method, etc [4]. The first three methods define the quantity and form of the surface hairiness through transition of physical indexes, while the latter one directly simulates the compression form when the fabric contacts skin to obtain relevant force value.

Ao Limin [3] has improved the low-stress compression measurement method, and developed a fabric single-side compression tester to measure mechanical properties of filament. According to the fiber needle bending model with one end fixed and the other end free, Qi Yuan [5] studied the axial compression bending property of fabric hairiness. On this basis, Liu Yuqing [6] adopted the multiple measurement ideology based on Euler compression bar model to design mechanical device and control software, which organically combined the force measurement with the form evaluation to characterize the puncture property of filament.

Berg mami et al [7-9] adopted the ranking method to rank varieties of stimulation in terms of roughness sense. Cholewiak and Louw, et al [10, 11] selected paired comparison method to analyze the threshold value and difference threshold value of roughness sensibility. Lederman et al [12-15] adopted quantity estimation method to study the influence factor of roughness sense and its psychophysical measurement attributions.

At present, the research on roughness sense of fabrics mainly adopts objective evaluation method, i.e., measuring the basic mechanical and physical property parameters related to roughness sense of the fabrics with instrument, and measuring and representing the intensity of roughness sense with mathematical statistics method and specific indicators. The kinds of instrument adopted include KES-F system, YG821 surface friction tester, UST general tester, micro-friction sensor, etc [16-18]. Ren Jian [19] designed a kind of new disk-method test system to optimize the test of surface roughness of fabrics.

Based on the analysis on the fabric contiguous sense evaluation methods and devices, the experimental 
paradigm of cognitive behaviors is introduced to study cognitive activities in the contiguous sense cognition of the tested linen fabrics. The experimental program is written with E-Prime 2.0, while the expression of the experimental stimulus material is realized with the self-made contiguous sense measurement device, which output the senses of itch, roughness and smoothness among the fabric contiguous sense, so as to test the feasibility of the proposed method.

\section{EXPERIMENTAL METHODS}

\section{A. Subjects}

Sixty-four young female undergraduates with relevant specialized knowledge are selected randomly as subjects, aged from 20 to 23 years. The reas on to choose female college students is that the study of Gamsworthy [20] showed that females were more sensitive than males. All the subjects are normal in vision or normal after correction, and they are right hander. All the subjects are separately measured, and have not done any similar experiment.

Gamsworthy [20] adopted forearm measurement method to conduct subjective evaluation on fabric tactile sensibility, and found that the skin on the forearms have excellent sensibility for external stimulation. Then, Naylor [21] adopted the method to conduct experiments on urtication sense, and further proved the feasibility and scientificity of arm evaluation method. Therefore, this experiment adopts forearm as measurement location.

\section{B. Design of dressing contiguous sense measurement device}

The self-made device comprises the base (1), main control system (2), and three-freedom-degree driving installation (3) fixed on the base, the measurement installation (4) set on the driving installation, whose location can be adjusted in $\mathrm{X}, \mathrm{Y}$ and $\mathrm{Z}$ coordinate space. Through the driving of the installation, the displacement with different speeds or forces can be realized, as shown in Fig. 1.

When using the device, open power switch of the main control system, and press start button on the main control system, so as to transmit the order to the stepper motor (33) through the stepper motor driver (34), and regulate the mechanical movement of the screw rod (32) at the three directions of $\mathrm{X}, \mathrm{Y}, \mathrm{Z}$, and conduct the zero correction of the measuring wheel (41).

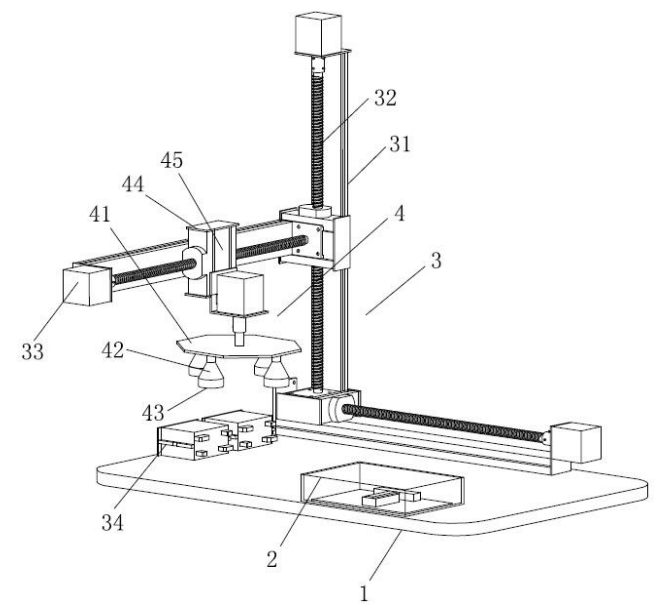

Figure 1. Self-made measurement device

In the behavioral experiment mode, the expression program of the experimental paradig $m$, the expression of experimental stimulation materials, the random display of evaluation indexes, and the storage program of evaluation results has been designed firstly. The experiment program is shown in Fig. 2. The experiment is conducted on computer, and its program is written with E-Prime2.0.

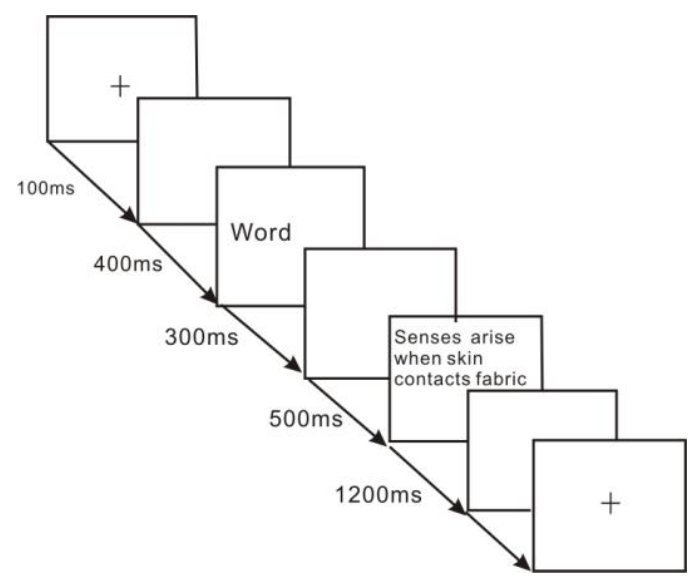

Figure 2. A schematic illustration of the trial

After the evaluation indexes show, the measuring head (42) loading fabric starts to contact the skin. When the contact is detected by the pressure sensor (43), the sensor will send the information to the main control system, which will send the order to control the stepper motor to run, and the fabric will start to have movement relative to the skin; when the proximity sensor (44) detects the specified route mark, it will give an information feedback to the main control system, which will send an order to stop the measuring wheel. During this process, the subjects feel tactile sensibility with the fabric, and makes quantization judgment. The continuous evaluation results are saved in the storage system.

After completion of one measurement, press the button on the main control system, then the measuring wheel will automatically rotate for $1 / 4$ circle, and the measuring wheel will move to the initial position, i.e., zero position, where the next fabric will contact the skin. 
The reaction data will be generated by the subjects through pressing buttons. If the subject feels that the fabric tactile sensibility and the meaning of sensory word is same and the sense is quite strong, press button "/"; if the subject feels the sense is common, press button " $X$ "; if the subject feels that fabric contiguous sense and the meaning of sensory word is different, press button " $Z$ ".

During the experiment, subjects could be given time to rest and everyone participated once in the same experiment to prevent memory mechanism disturbing subjects' cognitive processes

\section{RESULTS AND DISCUSSION}

\section{A. Device measurement of fabric contiguous sense}

Pure linen, cotton linen, and linen -viscose blending, etc. are deeply favored by consumers due to their properties such as good wear-ability and environmental protection, etc. $8 \mathrm{kinds}$ of fabric are randomly selected in an apparel enterprise, whose specification parameters are shown in Table1.

TABLE I. SPECIFICATION PARAMETERS OF LINEN FABRICS

\begin{tabular}{|c|c|c|c|c|c|c|c|}
\hline No. & Fabric weave & Ingredient & $\begin{array}{c}\text { Linear density } \\
\text { of warp yarn }\end{array}$ & $\begin{array}{c}\text { Linear density } \\
\text { of weft yarn }\end{array}$ & $\begin{array}{c}\text { Warp } \\
\text { density }\end{array}$ & $\begin{array}{c}\text { Weft } \\
\text { density }\end{array}$ & $\begin{array}{c}\text { Areal } \\
\text { density }\end{array}$ \\
\hline 1\# & Plain weave & L55/V45 & 29.53 te $\times 1$ & 42.18 te $\times 1$ & 59 & 52 & 156 \\
\hline 2\# & Plain weave & L100\% & 42.18 tex $\times 1$ & 42.18 te $\times 1$ & 54 & 52 & 165 \\
\hline 3\# & Plain weave & L55/C45 & 28.12 te $\times 1$ & 42.18 te $\times 1$ & 54 & 50 & 156 \\
\hline 4\# & Plain weave & L55/V45 & 59.05 te $\times 1$ & 59.05 tex $\times 1$ & 44 & 38 & 192 \\
\hline 5\# & Plain weave & L55/C45 & 53.68 te $\times 1$ & 53.68 tex $\times 1$ & 51 & 47 & 195 \\
\hline 6\# & Plain weave & L100\% & 42.18 tex $\times 1$ & 42.18 tex $\times 1$ & 54 & 54 & 165 \\
\hline 7\# & Plain weave & L55/C45 & 39.37 te $\times 1$ & 39.37 tex $\times 1$ & 54 & 52 & 165 \\
\hline 8\# & Plain weave & L55/V45 & 19.68 te $\times 2$ & 42.18 te $\times 11$ & 52 & 51 & 175 \\
\hline
\end{tabular}

In order to conduct a quantitative evaluation on contiguous senses, three-point system is adopted to assign each evaluation index. If the subject feels that the fabric contiguous sense and the meaning of sensory word is same and the sense is quite strong, the evaluation value will be 2 points; if the sense is quite common, the evaluation value will be 1 point; if the subject feels that the fabric contiguous sense is different from the meaning of sensory word, the evaluation value will be 0 point.

Variance analysis on evaluation values of the senses of urtication, roughness and smoothness of 8 kinds of fabric is done by using SPSS 20, and the analyzed results are shown in Table 2.

TABLE II. VARIANCE ANALYSIS RESULT

\begin{tabular}{|c|c|c|}
\hline Sensory word & F & P-value \\
\hline Urtication & 8.691 & 0.000 \\
\hline Roughness & 5.766 & 0.000 \\
\hline Smoothness & 7.026 & 0.000 \\
\hline
\end{tabular}

Table 2 shows that when expression of the stimulation material adopts self-made devise, the significance level of the senses of urtication, roughness and smoothness will be: $\mathrm{P}<0.05$. Therefore, the contiguous senses among $8 \mathrm{kinds}$ of fabrics have significant differences with each other.

In order to study the differences of the 8 fabrics in terms of those senses, SPSS 20 is employed to accomplish multiple comparisons analysis, and the results are shown in Table 3.
TABLE III. MULTIPLE COMPARISONS ANALYSIS OF TACTILE SENSIBILITY

\begin{tabular}{|c|c|c|c|c|}
\hline \multirow{2}{*}{ F. } & \multirow{2}{*}{ F. } & \multicolumn{3}{|c|}{ Sig } \\
\hline & & Urtication & Roughness & Smoothness \\
\hline \multirow{7}{*}{1} & 2 & $0.000 \star$ & 0.848 & 0.813 \\
\hline & 3 & 0.980 & 0.732 & 1.000 \\
\hline & 4 & $0.001 \star$ & 0.443 & 0.955 \\
\hline & 5 & $0.001 \star$ & 0.116 & $0.004 \star$ \\
\hline & 6 & $0.005 \star$ & 1.000 & 1.000 \\
\hline & 7 & $0.002 \star$ & 0.929 & 0.955 \\
\hline & 8 & 0.159 & 0.993 & 0.955 \\
\hline \multirow{7}{*}{2} & 1 & $0.000 \star$ & 0.848 & 0.813 \\
\hline & 3 & $0.002 \star$ & $0.032 \star$ & 0.566 \\
\hline & 4 & 0.995 & 0.999 & 1.000 \\
\hline & 5 & 0.995 & 0.929 & 0.431 \\
\hline & 6 & 0.944 & 0.974 & 0.699 \\
\hline & 7 & 0.980 & 1.000 & 1.000 \\
\hline & 8 & 0.372 & 0.999 & 0.127 \\
\hline \multirow{3}{*}{3} & 1 & 0.980 & 0.732 & 1.000 \\
\hline & 2 & $0.002 \star$ & $0.032 \star$ & 0.566 \\
\hline & 4 & 0.051 & $0.003 \star$ & 0.813 \\
\hline
\end{tabular}




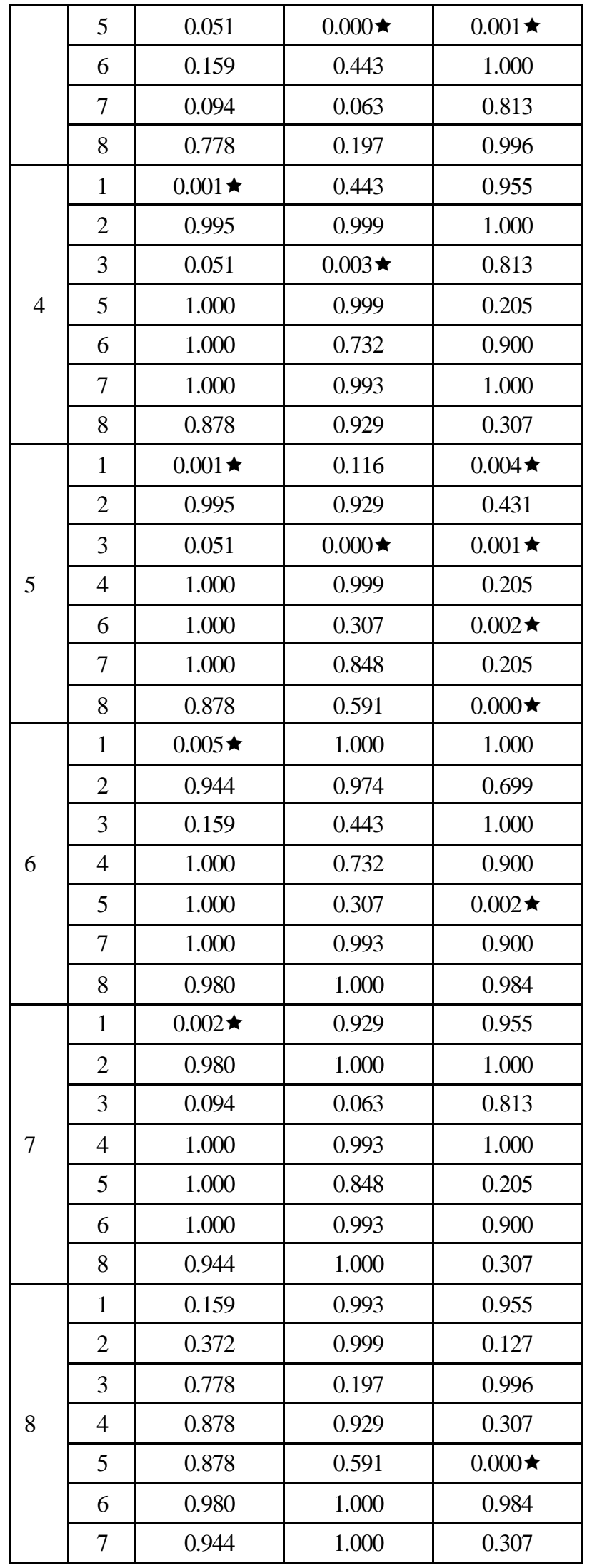

$\star$ The mean difference is significant at the 0.05 level

As shown in Table 3, from the perspective of the significance level of 0.05 , fabric 1 and fabric $2,4,5,6,7$ has obvious differences in terms of urtication sense; fabric 2 and fabric 3 has distinct differences in this sense.
The difference in urtication between fabric 1 and fabric 2, 6 is caused by that fabric 2,6 is pure linen fabric with grater linear density of warp yarn. When the fabric contact with human skin, the prominent linen fibers on the surface of fabric 2, 6 evoke intense stimulation to skin receptors, the perceptive urtrication is higher than that of fabric 1 . The difference between fabric 1 and fabric 4,5 is because that the linear density of warp yarn and weft yarn are greater than that of fabric 1. Exposed linen fiber in blending yarn will cause great stimulation to human skin due to larger initial modulus of linen fiber. The difference between fabric 1 and fabric 7 is caused by the difference in the linear density of warp yarn. There is significant difference between fabric 2 and fabric 3 because of the higher content of linen fiber and greater linear density of warp yarn of fabric 2 .

Fro $m$ the perspective of the significance level of 0.05 , Table 3 also indicates that fabric 2 and fabric 3 have significant differences in terms of roughness sense; fabric 3 and fabric 3, 4 have distinct differences in this sense.

The difference in roughness between fabric 2 and fabric 3 is caused by that fabric 2 is pure linen fabric with grater linear density of warp yarn. When the fabric contacts with human skin, the external stimulus will generate great influence towards receptors because of the properties of linen fiber, such as crosswise knur, great initial modulus and lower elastic recovery, then subject feel large roughness sense due to the enlarged discharge frequency and range. There is significant difference between fabric 3 and fabric 4, 5 because of the greater linear density of warp yarn of fabric 4,5 with rigid yarn.

It is clear that there are significant differences in smoothness sense between fabric 5 and fabric $1,3,6,8$. The main reason caused the difference is that fabric 5 with larger linear density of yarn and stiff hairiness on the surface.

\section{B. Feasibility test}

It is a common method for the expression of stimulus materials in the contiguous evaluation to let operators hold the fabrics in hands and contact the fabrics with the skin. The experiment has adopted the method to accomplish the evaluation of the senses of urtication, roughness and smoothness of 8 kinds of fabrics. Meanwhile, variance analysis is done by using SPSS 20, whose results are shown in Table 4.

TABLE IV. VARIANCE ANALYSIS RESULT BY MANUAL MEASUREMENT

\begin{tabular}{|c|c|c|}
\hline Sensory word & F & P-value \\
\hline Urtication & 4.168 & 0.000 \\
\hline Roughness & 3.804 & 0.001 \\
\hline Smoothness & 3.494 & 0.001 \\
\hline
\end{tabular}
experimental stimulus materials is measured by hand, the significance level of the senses of urtication, roughness and smoothness will be: $\mathrm{P}<0.05$. Therefore, the contiguous senses among 8 fabrics also have obvious differences with each other. 


\section{Comparative analysis}

The mean values of contiguous senses of urtication, roughness and smoothness after contacting the fabrics with skin are shown in Fig. 3, 4 and 5.

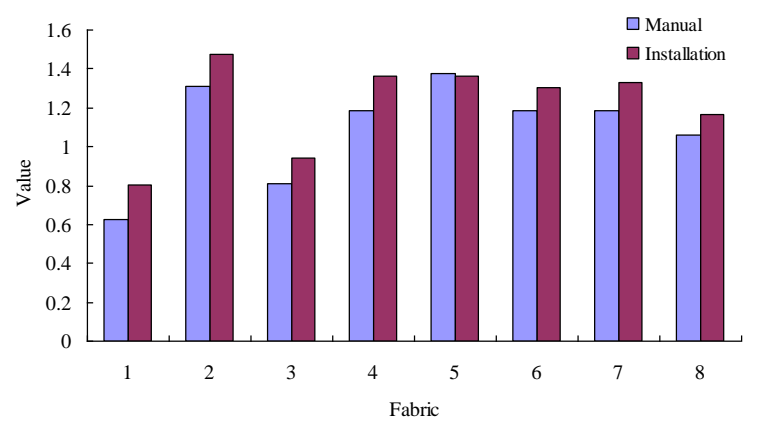

Figure 3. Evaluation value on urtication

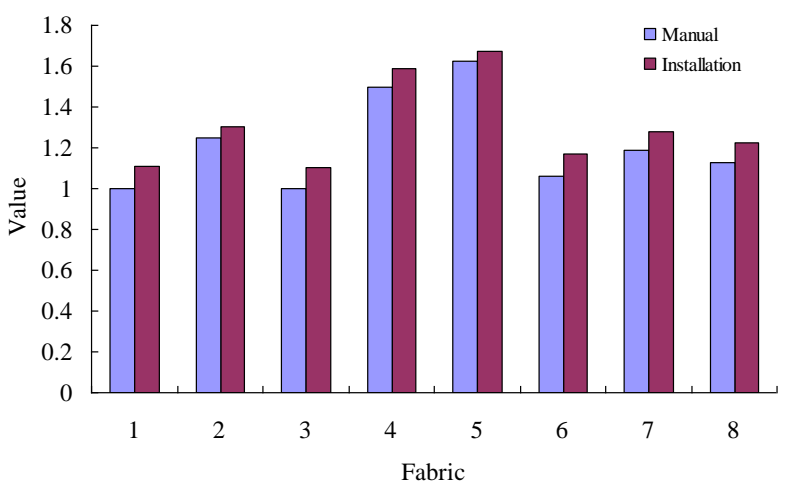

Figure 4. Evaluation value on roughness

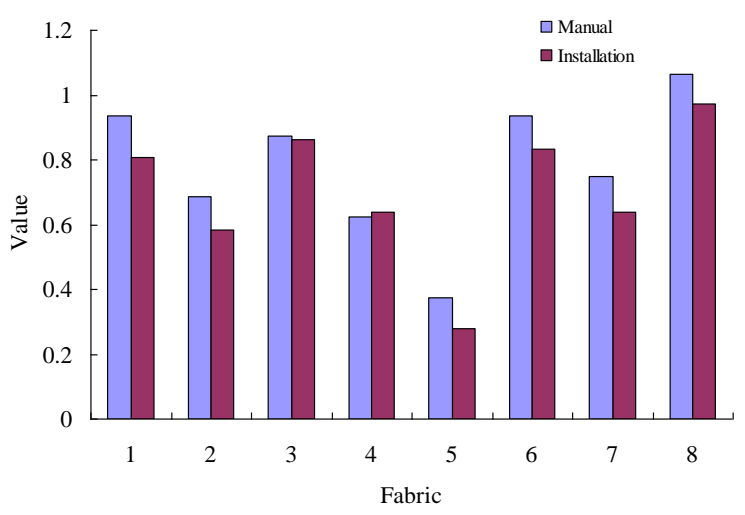

Figure 5. Evaluation value on smoothness

The results of Fig. 3 show that, when the expression of experimental stimulus materials is measured by hand or by device, the difference on evaluation values in terms of urtication sense will be quite small, and can well distinguish the intensity of urtication sense of those fabrics.
The results also indicate that, except for fabric 5\#, the evaluation values of urtication sense obtained from measurement by device is slightly higher than that measured by hand. The device has set with pressure sensor (thin film pressure sensor) on the lower surface of the measuring head, which has quite high sensitivity, and can well control contiguous pressure. And the device has constant moving speed. Therefore, comparing with manual method, it can realize constant pressure and more stable moving speed, good for the sufficient contact between the fabrics and skin. Garnsworthy [20] has found that mechanics excitation condition for a single fiber is that the bending deformation force of the fiber must be over $0.75 \mathrm{mN}$, or the human body will not feel the sense of urtication, but the value varies with each individual. In this paper, the minimum of the tactile force is $0.1 \mathrm{~N}$, and that much greater than threshold of untrication. Therefore, when the expression of experimental stimulus material adopts device measurement, evaluation results of the urtication sense will be more reasonable.

As shown in Fig. 4, when the expression of experimental stimulus material adopts manual measurement or device, the difference on evaluation values in terms of roughness sense will be quite s mall, and can well distinguish the intensity of roughness senses of the 8 kinds of fabrics, containing some fibrilia. Because the length and linear density of fibrilia have great discreteness, the fineness uniformity of the linen yarn is not so good. Under the force, yams easy to slip to enlarge the space of yarns. Therefore, the evaluation value of roughness sense obtained with device method is slightly higher.

When the fabric moves on the surface the human skin, it increases the deformation of skin to generate the roughness sensation. The friction and mechanical reaction between fabric and human skin are the main factors for the generation of roughness sense. When the self-made device is employed to express the stimulus material, the tactile force from fabric and human skin is even, which evokes the friction and mechanical reaction directly, and induces responses of mechanical receptors fully. So the perceived roughness sense of subject is larger than that of manual measurement.

Fig. 5 showed that when the expression of experimental stimulus material adopts manual measurement or device, the difference on the evaluation values in terms of smoothness sense will be quite small, and can well distinguish the intensity of smoothness sense of those fabrics. When device measurement is adopted, evaluation values of both urtication sense and roughness sense will be slightly higher than that measured by hand, so the evaluation value of smoothness sense measured with installation will be slightly lower. The results of Fig. 5 have indicated the consistency between theoretically predicted results and actually evaluated results.

In order to further test the feasibility of adopting device evaluation for expression of experimental stimulus materials, the results of $T$ test of the two evaluation values are shown in Table 5. 
TABLE V. T TEST ON INSTALLATION AND MANUAL MEASUREMENT

\begin{tabular}{|c|c|c|c|c|c|c|c|c|}
\hline $\begin{array}{c}\text { Sensory } \\
\text { word }\end{array}$ & Fabric & $\begin{array}{l}\text { T-value } \\
\text { (2-tialed) }\end{array}$ & $\begin{array}{c}\text { Sensory } \\
\text { word }\end{array}$ & Fabric & $\begin{array}{c}\text { T-value } \\
\text { (2-tialed) }\end{array}$ & $\begin{array}{c}\text { Sensory } \\
\text { word }\end{array}$ & Fabric & $\begin{array}{l}\text { T-value } \\
\text { (2-tialed) }\end{array}$ \\
\hline \multirow{8}{*}{ Urtication } & $1 \#$ & 0.363 & \multirow{8}{*}{ Roughness } & $1 \#$ & 0.592 & \multirow{8}{*}{ Smoothness } & $1 \#$ & 0.534 \\
\hline & 2\# & 0.409 & & 2\# & 0.785 & & 2\# & 0.646 \\
\hline & $3 \#$ & 0.532 & & $3 \#$ & 0.621 & & $3 \#$ & 0.953 \\
\hline & $4 \#$ & 0.450 & & 4\# & 0.584 & & $4 \#$ & 0.945 \\
\hline & $5 \#$ & 0.939 & & $5 \#$ & 0.433 & & $5 \#$ & 0.493 \\
\hline & $6 \#$ & 0.602 & & $6 \#$ & 0.542 & & $6 \#$ & 0.603 \\
\hline & $7 \#$ & 0.451 & & $7 \#$ & 0.650 & & $7 \#$ & 0.574 \\
\hline & $8 \#$ & 0.632 & & $8 \#$ & 0.570 & & $8 \#$ & 0.708 \\
\hline
\end{tabular}

As shown in Table 5, it is clear that the values of sig. (2-tialed) are higher than 0.05 , i.e., $\mathrm{P}>0.05$; it means that the contiguous evaluation values obtained from both experimental methods have no significant differences. Therefore, it is scientific and reasonable to adopt the device method for the expression of experimental stimulation materials. That is to say, the contiguous sense measurement method proposed in the paper is feasible.

\section{CONCLUSIONS}

In summary, the experimental paradig $m$ of cognitive behavior is introduced to study the cognitive activities of the subjects in the contiguous sense cognition of linen fabrics. The expression of experimental stimulation materials is mainly realized with self-made contiguous sense measurement device. Some conclusions can be obtained.

(1) When the expression of experimental stimulus material adopts manual measurement or device, the difference on evaluation values in terms of urtication, roughness and smoothness sense will be significant, and can well distinguish the intensity of the senses of urtication, roughness and smoothness.

(2) The evaluation values in terms of contiguous sense of fabrics obtained with manual measurement and device for the expression of experimental stimulation materials have no significant difference. The results indicate that the self-made contiguous sense measurement device combined with the introduction of behavioral experimental paradig $\mathrm{m}$ is feasible, presenting the contiguous sense of linen fabrics effectively.

\section{ACKNOWLEDGEMENT}

This paper is funded by the Dr Scientific Research Fund (No.Jit-b-201513).

\section{REFERENCES}

[1] L Yi; Comfort of Clothing and Product Design, China Textile\& Apparel Press, Beijing, 2002, pp.2-23.

[2] K B Franziska, F Berthold, "Psychophysics and Perceiving Granularity," Symposium on Haptic Interfaces for Virtual Environment and Teleoperator Systems, Alexandria, pp.387-393, 2006.

[3] A Limin, Yu Chongwen, "Sense Estimation and Instrumental Evaluation of Fabric-evoked Prickle," Journal of Donghua
University, vol. 21, Dec. 2004, pp. 82-87, doi: 10.3969/j.issn. 1672 $-5220.2004 .03 .017$

[4] M Matsudaira, J Watt, G Carnaby, "Measurement of the Surface Prickle of Fabrics, Part1: The Evaluation of Potential Objective Methods," Journal of the Textile Institute, vol. 81, 1990, pp.288-299.

[5] Q Yuan, Y Weidong, "Study on Evaluation of Urtication of Fabric," Journal of Qingdao University, vol.20, 2005, pp. 44-48.

[6] L Yuqing, Characterization for Fabric Tactile Perception based on Functional Magnetic Resonance Imaging and Meso-mechanic Analysis, Shanghai: Donghua University, 2009,pp.126-133.

[7] M Wouter, M Astrid, "Analysis of Haptic Perception of Materials by Multidimensional Scaling and Physical Measurements of Roughness and Compressibility," Acta Psychol, vol. 121, 2006, pp.1-20, doi:10.1016/j.actpsy.2005.04.005.

[8] M Wouter, $M$ Astrid, "Haptic and Visual Perception of Roughness," Acta Psychol, vol. 124, 2007, pp.177-189, doi:10.1016/j.actpsy.2006.03.002.

[9] P Jangwoon, H Wongi, J Jeongrim, "Development of a Textile Sensibility Evaluation System," Fibers and Polymers, vol. 14, Aug. 2013, pp. 1400-1406, doi: 10.1007/s12221-013-1400-0.

[10] A Steven, K Kwangtaek, T Hong, "A Frequency-domain Analysis of Haptic Grat ings," IEEE Transactions on Haptics, vol. 3, 2010, pp. 3-14, doi:10.1109/TOH.2009.36.

[11] S Louw, M Kappers, J Koenderink, "Haptic Detection of Sine-wave Gratings," Perception, vol. 34, 2005, pp. 869-885.

[12] L Roberta, L Susan, H Cherry, "Feeling Textures through a Probe: Effects of Probe and Surface Geometry and Exploratory Factors," Attention, Perception \& Psychophysics, vol. 65, 2003, pp.613-631, doi: 10.3758/BF03194587.

[13] K Roberta, L Susan, "Tactile Roughness Perception with a Rigid Link Interposed between Skin and Surface," Perception \& Psychophysics, vol.61, Jan 1999, pp.591-607, doi: $10.3758 / \mathrm{BF} 03205532$.

[14] O Troynikov, $\mathrm{N}$ Nawaz, $\mathrm{S} \mathrm{Vu}$, "Evaluation of Surface Characteristics of Inner Layer Fabrics Suitable for Structural Firefighters' Clothing," Textile Research Journal, vol. 82, 2012, pp.1014-1025, doi:10.1177/0040517511431312.

[15] S Les, C Armand, W Carole, "Identification of the most Significant Comfort Factors for Textiles from Processing Mechanical, Handfeel, Fabric Construction, and Perceived Tactile Comfort Data," Textile Research Journal, vol 83, 2013, pp.34-43, doi: $10.1177 / 0040517512438121$.

[16] P Deng, Research on the Fabric Roughness from the Surface Texture Composition, Shanghai: Donghua University, 2011, pp.12-13.

[17] F Hammond, R Kramer, Q Wan, "Soft Tactile Sensor Arrays for Force Feedback in Micromanipulation," IEEE Sensors Journal, vol.14, 2014,pp.1443-1452, doi: 10.1109/JSEN.2013.2297380. 
[18] G Michele, M Maura, P Margherita, "Electro-tactile Device for Material Texture Simulation," Int J Adv Manuf Technol, vol.68, Oct. 2013, pp. 2185-2203, doi: 10.1007/s00170-013-4832-1.

[19] R Jian, Measurement based on Wavelet Transform for Testing Fabric Frictional Properties by a Rotating Method, Shanghai: Donghua University, 2008, pp.11-46.
[20] K Garnsworthy, L Gully, P Kenins, "Transcutaneous Electrical Stimulation and the Sensation of Prickle," J Neurophysiology, vol. 59, 1988, pp.1116-1127.

[21] G Naylor, "The Coarse Fiber Component and Fiber end Diameter Characteristics of Australian Wool Tops," International Journal of Sheep and Wool Science, vol. 40, 1992, pp.14-18.doi: $10.2527 /$ jas. 2007-0109. 